\title{
Random Noise Stimulation Improves Neuroplasticity in Perceptual Learning
}

\author{
Anna Fertonani, ${ }^{1 \star}$ Cornelia Pirulli, ${ }^{1 \star}$ and Carlo Miniussi ${ }^{1,2}$ \\ ${ }^{1}$ Cognitive Neuroscience Section, IRCCS Centro San Giovanni di Dio Fatebenefratelli, 25125 Brescia, Italy, and ${ }^{2}$ Department of Biomedical Sciences and \\ Biotechnologies, National Institute of Neuroscience, University of Brescia, 25123 Brescia, Italy
}

Perceptual learning is considered a manifestation of neural plasticity in the human brain. We investigated brain plasticity mechanisms in a learning task using noninvasive transcranial electrical stimulation ( $\mathrm{tES}$ ). We hypothesized that different types of tES would have varying actions on the nervous system, which would result in different efficacies of neural plasticity modulation. Thus, the principal goal of the present study was to verify the possibility of inducing differential plasticity effects using two tES approaches [i.e., direct current stimulation (tDCS) and random noise stimulation (tRNS)] during the execution of a visual perceptual learning task.

One hundred seven healthy volunteers participated in the experiment. High-frequency tRNS (hf-tRNS, $100-640 \mathrm{~Hz}$ ), low-frequency tRNS (lf-tRNS, 0.1-100 Hz), anodal-tDCS (a-tDCS), cathodal-tDCS (c-tDCS), and sham stimulation were applied to the visual areas of the brain in a group of volunteers while they performed an orientation discrimination task. Furthermore, a control group was stimulated on the vertex $(\mathrm{Cz})$. The analysis showed a learning effect during task execution that was differentially modulated according to the stimulation conditions. Post hoc comparisons revealed that hf-tRNS significantly improved performance accuracy compared with a-tDCS, c-tDCS, sham, and $\mathrm{Cz}$ stimulations.

Our results confirmed the efficacy of hf-tRNS over the visual cortex in improving behavioral performance and showed its superiority in comparison to others tES. We concluded that the mechanism of action of tRNS was based on repeated subthreshold stimulations, which may prevent homeostasis of the system and potentiate task-related neural activity. This result highlights the potential of tRNS and advances our knowledge on neuroplasticity induction approaches.

\section{Introduction}

Perceptual learning (PL) is a form of implicit memory that is characterized by an improvement in sensory discrimination after repeated exposure to particular types of stimuli (Fahle and Poggio, 2002; Thiele, 2004). PL is considered a manifestation of neural plasticity (Gilbert et al., 2001; Li et al., 2004; Carmel and Carrasco, 2008). Thus, the study of the neural changes associated with PL can increase our understanding on neuroplasticity induction in adult sensory cortices.

Noninvasive transcranial electrical stimulation (tES) seems particularly promising for the induction and investigation of plasticity effects. Transcranial direct current stimulation (tDCS) is a type of tES that modulates cortical excitability in a polaritydependent manner. Generally, anodal stimulation increases neural activity, whereas cathodal stimulation decreases neural activity (Nitsche et al., 2008). Recently, interest has developed in

Received April 21, 2011; revised Aug. 23, 2011; accepted Sept. 5, 2011.

Author contributions: A.F., C.P., and C.M. designed research; A.F. and C.P. performed research; A.F. and C.P. analyzed data; A.F., C.P., and C.M. wrote the paper.

This research was supported by a Project Grant from the "Ministero della Salute-Ministry of Health" and from Associazione Fatebenefratelli per la Ricerca. C.M. was also supported by the James S. McDonnell Foundation.

${ }^{*}$ A.F. and C.P. contributed equally to this work.

The authors declare no competing financial interests.

Correspondence should be addressed to Carlo Miniussi, Department of Biomedical Sciences and Biotechnologies, University of Brescia, Viale Europa 11, 25123 Brescia, Italy. E-mail: carlo.miniussi@cognitiveneuroscience.it.

DOI:10.1523/JNEUROSCI.2002-11.2011

Copyright $\odot 2011$ the authors $\quad 0270-6474 / 11 / 3115416-08 \$ 15.00 / 0$ transcranial random noise stimulation (tRNS), which consists of the application of an alternating current over the cortex at random frequencies $(0.1-640 \mathrm{~Hz})$. Terney et al. (2008) demonstrated that tRNS over the motor area positively modulates cortical excitability and improves motor learning. Long-term potentiation (LTP) has been postulated as a likely mechanism underlying these effects (Nitsche et al., 2009); nevertheless, most tES studies have been based on the motor system (Nitsche et al., 2008).

In the present study, we aimed to test the effectiveness of tES techniques on the primary visual cortex (V1) by applying tES while healthy subjects executed an orientation discrimination task that involved V1 (Schoups et al., 2001). In particular, we evaluated the effects of two excitatory stimulations, expected to induce facilitatory effects at behavioral level, tRNS and anodal tDCS, on visual PL. Although previous studies have shown that tRNS and anodal tDCS had similar effects on the motor system (Nitsche et al., 2003a; Terney et al., 2008; Moliadze et al., 2010), our hypothesis was that these two different stimulation protocols would have different effects on visual system. We hypothesized that tRNS would be more effective in inducing behavioral improvement than tDCS for two reasons. First, because tRNS is a repetitive stimulation, it may induce direct temporal summation of neural activity, which occurs when the time constant of a neuron is sufficiently long to permit the summation of two stimuli presented in close sequence. This effect would cause the stimulated neurons to approach earlier their response threshold (Ter- 
ney et al., 2008). Second, tRNS may not induce the homeostatic phenomena of ion neural channels because the electrical field in which they are embedded constantly changes in an alternating random-frequency mode. On the contrary, with tDCS the neurons are embedded in a constant electrical field, which should allow the membrane responses to adapt and return to an initial "resting" state.

Therefore, we expected to observe greater performance improvement with high-frequency tRNS (hf-tRNS) than with anodal tDCS (a-tDCS), cathodal tDCS (c-tDCS), and sham stimulation. We also tested the specificity of hf-tRNS effects in two additional experiments: by applying hf-tRNS to a control site (i.e., the vertex, Cz in the 10-20 International EEG system) and by applying hf-tRNS after a baseline block (i.e., without stimulation).

\section{Materials and Methods \\ Subjects}

One hundred seven healthy subjects took part in the experiment. All of the participants were right handed with normal or corrected-to-normal vision. We did not include subjects with a history of seizures, implanted metal objects, heart problems, or any neurological disease. Moreover, as a standard procedure, subjects who did not reach an established learning performance in the task were excluded from the study; based on this criteria, 8 participants were excluded. The remaining 99 subjects were divided as follows: 6 participants $(2$ males, mean age \pm SD $35.0 \pm 7.2$ years; range $29-48$ years) were tested in a pilot behavioral experiment, 84 participants ( 42 males, mean age $21.7 \pm 2.5$ years; range $19-30$ years) took part in the main experiment, and 9 participants ( 3 males, mean age $31.7 \pm 3.9$ years; range $24-38$ years) were tested in a control hf-tRNS experiment. The 84 subjects participating in the main experiment were divided into six groups. All groups had 14 participants $(7$ males and 7 females), and the mean ages of the groups were as follows: $22.4 \pm 2.8$ years for hf-tRNS group, $21.7 \pm 2.9$ years for lf-tRNS group, $21.8 \pm$ 2.3 years for a-tDCS group, $21.7 \pm 2.6$ years for $\mathrm{c}$ - $\mathrm{tDCS}$ group, $21.6 \pm 3.0$ years for sham group, and $20.9 \pm 1.6$ years for the Cz group, respectively.

The present study was approved by the Ethics Committee of the Istituto di Ricovero e Cura a Carattere Scientifico San Giovanni di Dio Fatebenefratelli, Brescia, Italy. Safety procedures based on noninvasive brain stimulation approaches were adopted (Poreisz et al., 2007; Rossi et al., 2009), and informed consent was obtained from all participants before the beginning of the experiment.

\section{Ovarian hormone influence of data variability}

The influence of ovarian hormones on task performance has been demonstrated in many transcranial magnetic stimulation studies (Smith et al., 1999, 2002; Inghilleri et al., 2004; Sale et al., 2007). Indeed, these studies have shown that the cortical excitability of male and female subjects is only similar during the follicular phase of the menstrual cycle when progesterone levels are low and estrogen levels are high (Inghilleri et al., 2004). Furthermore, tDCS studies (Kuo et al., 2006; Chaieb et al., 2008) have highlighted gender differences in both visual and motor domains. These differences are likely due to hormonal effects, but no studies have controlled for this factor. The female participants in the present study were tested during the follicular menstrual phase (mean day from the first day of the menstrual period: $14.1 \pm 3.0$, range 9-20 d), because this is the period when progesterone levels are low and estrogen levels are high. Under these conditions, the cortical excitability of male and female subjects was similar (Inghilleri et al., 2004).

\section{Orientation discrimination task}

We chose the orientation discrimination task (ODT) because it is a widely studied visual perceptual learning task (Vogels and Orban, 1985; Shiu and Pashler, 1992) that involves V1 neurons. Many studies have demonstrated that V1 cells are highly specific for basic stimulus characteristics, such as orientation and direction (Ts'o et al., 1986; Schiltz et al., 1999; Schoups et al., 2001; Yacoub et al., 2008).

In the ODT task, participants had to decide whether the presented stimulus was tilted clockwise or counterclockwise relative to the previ-

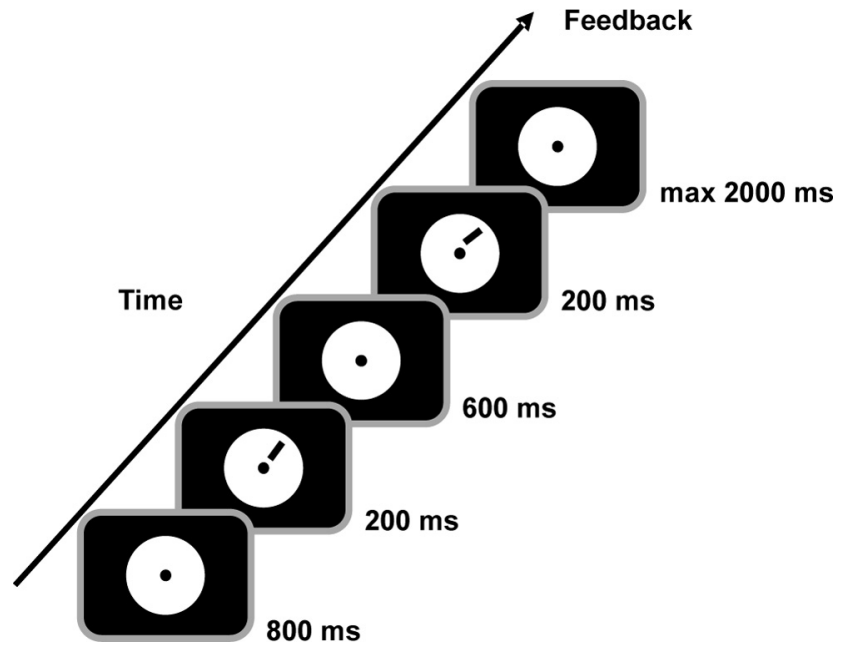

Figure 1. Trial structure. Example of an orientation discrimination task trial with the reference and target stimuli presented in the upper right hemifield.

ously presented stimulus. All stimuli were black lines, and each line stimulus was $2^{\circ}$ long and $5^{\prime}$ wide (in visual angle). The orientation of the reference stimulus was $45^{\circ}$ in the upper right and lower left hemifields and $135^{\circ}$ in the upper left and lower right hemifields. The angular differences between the reference and the target stimulus were 1.1, 1.21, 1.33, and $1.46^{\circ}$ (Matthews et al., 1999). The reference was presented first in half of the trials and second in the other half of the trials. All of experimental parameters just mentioned were balanced and randomized between blocks. The trial structure is described in Figure 1. The subjects were asked to respond as quickly and accurately as possible after the second stimulus was presented by pressing the left (counterclockwise) or right (clockwise) button of a response pad with the left or right index finger, respectively. Auditory feedback (duration $=50 \mathrm{~ms}$; frequency for the correct response $=700 \mathrm{~Hz}$; frequency for the incorrect response $=350$ $\mathrm{Hz}$ ) informed the subjects about the correctness of their responses.

Stimuli were presented on a computer screen using Presentation software v. 12.0 (http://www.neurobs.com) in each of the four visual hemifields: upper left, upper right, lower left, and lower right. In each trial, the two stimuli were presented in the same hemifield (Fig. 1). To limit the area in which the stimuli were presented, a black piece of cardboard covered the screen except for a circle that was $10 \mathrm{~cm}$ in diameter located at the center of the screen. A central fixation point was maintained for the duration of the trial.

In the main experiment, each block of the ODT consisted of 64 trials and lasted $\sim 4 \mathrm{~min}$. The ODT consisted of eight blocks plus a training block. The training block was similar to the trial blocks, but it had a different number of trials (only 8 ) and an increased rotation angle between the two stimuli ( $15^{\circ}$ clockwise or counterclockwise). The last block (i.e., the eighth one) was a fictitious block that only consisted of 16 trials.

\section{Pilot experiment}

The pilot experiment aimed to determine the ideal parameters of the ODT. The subjects were asked to complete five blocks of 128 trials each, and the results are presented in Table 1 . All of the subjects reported a sense of fatigue at the end of each of the five blocks, which was more pronounced for the last two blocks. For this reason, eight shorter blocks (i.e., each block contained half as many trials as the original block) were presented in the main experiment.

\section{Main experiment}

Stimulation techniques: $t R N S$ and tDCS. The stimulations were delivered by a battery-driven stimulator (Eldith-Plus, NeuroConn) through a pair of saline-soaked sponge electrodes. The tRNS consisted of an alternating current of $1.5 \mathrm{~mA}$ intensity with a $0 \mathrm{~mA}$ offset applied at random frequencies. The frequencies ranged from 0.1 to $100 \mathrm{~Hz}$ for lf-tRNS, or from 100 to $640 \mathrm{~Hz}$ for hf-tRNS. The intensity of stimulation did not induce a 
Table 1. Pilot experiment: results of the orientation discrimination task

\begin{tabular}{|c|c|c|c|c|c|}
\hline & Block 1 & Block 2 & Block 3 & Block 4 & Block 5 \\
\hline Accuracy $d^{\prime}( \pm S D)$ & $0.07 \pm 0.35$ & $0.31 \pm 0.34$ & $0.50 \pm 0.19$ & $0.57 \pm 0.15$ & $0.61 \pm 0.16$ \\
\hline RTs (ms) ( \pm SD) & $693 \pm 134$ & $608 \pm 124$ & $589 \pm 120$ & $556 \pm 132$ & $545 \pm 125$ \\
\hline
\end{tabular}

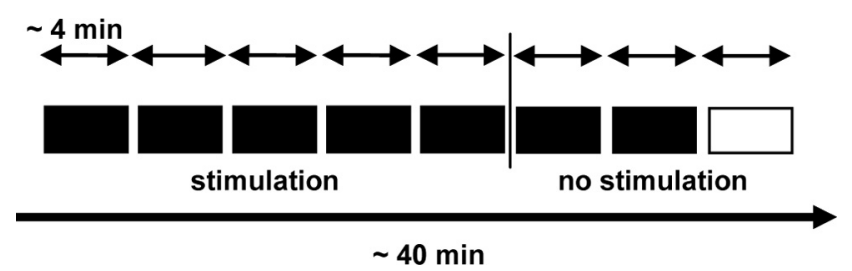

Figure 2. Experimental procedure. The main experiment was designed to be between subjects with six stimulation conditions: hf-tRNS, If-tRNS, a-tDCS, c-tDCS, sham, and Cz. Subjects received the stimulation only in the first five blocks. The last block was a fictitious block (white represented); data from this block were not analyzed.

phosphene perception in either of the frequency bands (Kanai et al., 2008). The tDCS consisted of a direct $1.5 \mathrm{~mA}$ current.

In the main experiment, the stimulations were applied for $\sim 4 \mathrm{~min}$ during each of the first five experimental blocks. The total duration of the stimulations was $\sim 22 \mathrm{~min}$. The active electrode had an area of $16 \mathrm{~cm}^{2}$, whereas the reference had an area of $60 \mathrm{~cm}^{2}$. The current density was maintained below the safety limits (varying between 25 and $60 \mu \mathrm{A} / \mathrm{cm}^{2}$ ) (Poreisz et al., 2007). The electrodes were kept in place with elastic bands, and an electroconductive gel was applied under the electrodes before the montage to reduce skin impedance. The active electrode was applied over the occipital cortex. When tDCS stimulation was applied, the polarity of the active electrode was anodal in the a-tDCS condition and cathodal in the c-tDCS condition. The individual target area was determined by examining the position in accordance with the 10-20 International EEG system. This procedure was performed starting at $10 \%$ of the nasioninion distance above the inion. The mean position for $\mathrm{V} 1$ stimulation was determined to be $3.5 \pm 0.2 \mathrm{~cm}$ above the inion. The reference electrode was fixed extracephalically on the right arm. In the sham stimulations, the current was turned off $20 \mathrm{~s}$ after the stimulation began (Gandiga et al., 2006). Additionally, we used a control condition to test the specificity of the hf-tRNS effects by administering active stimulation over the vertex, which is a brain area that is not involved in our task. All of the stimulation parameters were identical to hf-tRNS except for the active electrode, which was placed on the $\mathrm{Cz}$ location.

At the end of the experimental session, we asked all subjects to complete a questionnaire developed by our research laboratory about the sensations they experienced during the different stimulations (Fertonani et al., 2010).

Procedure. The participants were seated in front of a computer screen in a quiet, semidark room. A $57 \mathrm{~cm}$ distance from the screen was maintained through the use of a chin rest. The experiment was a betweensubjects design with six stimulation conditions: hf-tRNS, lf-tRNS, a-tDCS, c-tDCS, sham stimulation, and Cz.

The subjects began the ODT $10 \mathrm{~s}$ after the onset of the stimulation. The procedure is depicted in Figure 2.

In the real stimulation conditions (hf-tRNS, lf-tRNS, a-tDCS, c-tDCS, and $\mathrm{Cz}$ ), the stimulation was only delivered during the first five blocks of the task. In the last three blocks, sham stimulation was applied for $20 \mathrm{~s}$ at the beginning of each block. In the sham condition, the stimulation was a placebo for all eight blocks and was delivered for $20 \mathrm{~s}$ at the beginning of each block. The duration of the entire experimental session was $\sim 45 \mathrm{~min}$.

Data analysis. The average orientation sensitivity was calculated in terms of a $d^{\prime}$ value for each subject and each block, separately for each stimulation condition. Because the ODT is a two-alternative forcedchoice task, a value of $d^{\prime}=1$ corresponded to a $75 \%$ accuracy rate.

As an index of learning rate, we analyzed the relationship between $d^{\prime}$ values and the log transformation of block numbers using linear regression analysis. This analysis allowed us to associate a slope value with each subject.
We also analyzed the mean reaction times (RTs) of the correct response trials for each subject and each block.

For all data ( $d^{\prime}$ values, slopes, and RTs), the Kolmogorov-Smirnov test confirmed the normality of the distribution, and the data were analyzed using a repeated-measures ANOVA. The data sphericity was tested using the Mauchly test where appropriate. When the test results were statistically significant, the data were corrected using the Huynh-Feldt correction. A $p$ value $<0.05$ was considered significant for all statistical analyses. For multiple comparisons, we used Fisher's least significant difference method to test our specific "a priori" hypotheses (i.e., to compare the different stimulation conditions). For all other comparisons, the $p$ values were corrected using a Bonferroni correction.

Data from the sensations induced by tES were analyzed using a oneway ANOVA for each sensation to compare the different stimulation types. The $p$ values were corrected using Bonferroni corrections. In addition, we calculated an intensity of perceived sensations index for each subject (i.e., the sum of the values reported in the questionnaire for each sensation) and correlated it with the mean $d^{\prime}$ values in the five blocks of real stimulation.

\section{Control-hf-tRNS experiment}

In the main experiment, we found that hf-tRNS applied over V1 improves the performance in the ODT. This effect is immediately evident (i.e., from the first block, see Fig. 3) and persists until the end of the stimulation, differently from a-tDCS. To confirm that this initial effect was due to the stimulation, and not to baseline differences across groups, we tested an additional group of subjects in an experimental design that included an initial baseline block without stimulation.

In this experiment, the participants executed six blocks of the ODT. The first block was without stimulation, while in the following five, hftRNS was applied over V1. In this way, all the parameters of the task and of the stimulation were maintained as in the main experiment, except for the moment in which the stimulation was applied (first vs second block).

\section{Results}

\section{Orientation sensitivity- $d^{\prime}$}

We performed a repeated-measures ANOVA with Block (from 1 to 7 ) as a within-subjects factor and Stimulation Condition (hf-tRNS, lf-tRNS, a-tDCS, c-tDCS, sham, and Cz) and Gender (male and female) as between-subjects factors. We observed a significant main effect for Block $\left(F_{(6,432)}=29.34 ; p<\right.$ $0.001)$, Stimulation Condition $\left(F_{(5,72)}=3.49 ; p<0.01\right)$, and Gender $\left(F_{(1,72)}=6.82 ; p<0.05\right)$. Regarding the main effect of Block, multiple post hoc comparisons revealed a statistically significant difference between block 1 and blocks $3,4,5,6$, and 7; between block 2 and blocks 4, 5, 6, and 7; between block 3 and blocks 6 and 7; and between blocks 4 and 7 .

Regarding the main effect of Stimulation Condition, multiple post hoc comparisons revealed that hf-tRNS (mean $d^{\prime} \pm \mathrm{SEM}=$ $0.770 \pm 0.130)$ was significantly different from sham $(0.375 \pm$ $0.116)$, a-tDCS $(0.509 \pm 0.134), \mathrm{c}-\mathrm{tDCS}(0.377 \pm 0.125)$, and $\mathrm{Cz}$ $(0.376 \pm 0.119)$ conditions (Fig. 3$)$. The difference between hftRNS and lf-tRNS $(0.617 \pm 0.124)$, however, was not significant $(p=0.220)$, and the difference between lf-tRNS and sham stimulation was marginally significant $(p=0.053)$. The Gender factor was statistically significant. Males were more accurate than females in all conditions (males: $0.597 \pm 0.125$; females: $0.411 \pm$ 0.131 ). The absence of any interaction between the Gender and Stimulation Condition factors, however, discouraged us from examining the gender factor in more detail. 


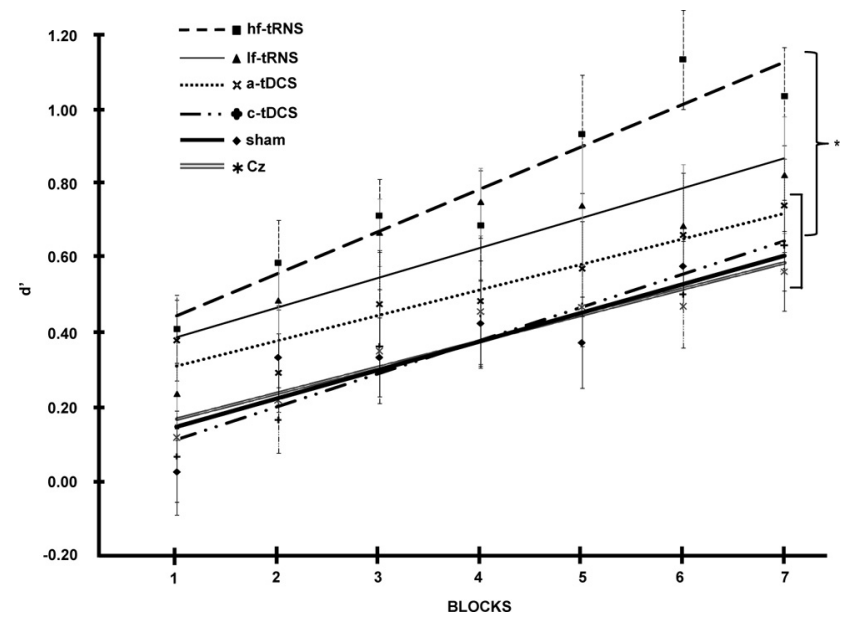

Figure 3. Main experiment results. Data are represented as the mean \pm SEM. The lines represent the fit of each condition: the broken line corresponds to hf-tRNS, the continuous thin line corresponds to If-tRNS, the dotted line represents a-tDCS, the mixed dotted-broken line represents c-tDCS, the continuous thick line corresponds to sham, and the double gray line represents C $z$ stimulation. The asterisk near the curly bracket indicates the statistically significant differences between hf-tRNS and the conditions in the square bracket $(p<0.05)$.

Repeated-measures ANOVA of each stimulation condition in the five blocks with real stimulation highlighted the main effect of Block $(p<0.01)$ for all stimulation conditions except a-tDCS. Post hoc comparisons in the hf-tRNS condition revealed that block 1 was different from blocks $3,4(p=0.065)$, and 5, and block 2 differed from block 5 . In the lf-tRNS condition, block 1 was different from blocks 3, 4, and 5. In the c-tDCS condition, block 1 was different from blocks 4 and 5 , and block 2 was different from block 5 . In the sham and $\mathrm{Cz}$ conditions, block 1 was different from blocks 4 and 5. No statistically significant differences were observed in the a-tDCS condition, which suggested the absence of a learning effect during a-tDCS.

To evaluate the stimulation effects at different time points of the protocol (blocks 1 and 5: beginning and end of stimulation, respectively; blocks 6 and 7: after the stimulation ended), we compared the different stimulations by separately considering each block. In block 1 , there was a significant main effect of the Stimulation Condition $\left(F_{(5,78)}=2.58\right.$; $\left.p<0.05\right)$. Post hoc comparisons revealed differences between the hf-tRNS and the sham, $\mathrm{c}$-tDCS, and $\mathrm{Cz}$ conditions $(p<0.05)$ and between the a-tDCS and the sham and c-tDCS conditions $(p<0.05)$. In block 5 , there was a significant main effect of the Stimulation Condition $\left(F_{(5,78)}=2.42 ; p<0.05\right)$. Post hoc comparisons revealed a difference between the hf-tRNS and a-tDCS, sham, $\mathrm{c}$-tDCS, and $\mathrm{Cz}$ conditions $(p<0.05)$. In block 6 , after the stimulation ended, there was a significant main effect of the Stimulation Condition $\left(F_{(5,78)}=2.89 ; p<0.05\right)$. Post hoc comparisons revealed differences between the hf-tRNS and all other conditions $(p<0.05)$. In block 7 , only a marginally significant effect of the Stimulation Condition $\left(F_{(5,78)}=1.99 ; p=0.09\right)$ was present. Post hoc comparisons revealed a difference between the hf-tRNS and the sham, $\mathrm{c}$-tDCS, and $\mathrm{Cz}$ conditions $(p<0.05)$.

The present data support the initial hypothesis that tRNS was more efficacious than a-tDCS (measured by improvement in the subjects' performance). Among the excitatory stimulations, this result confirms that hf-tRNS had the most prominent effect.

\section{Learning rate-slopes}

To specifically evaluate learning rate differences for hf-tRNS and a-tDCS, the two main types of stimulation that increase cortical excitability, we compared learning performance during hf-tRNS and a-tDCS. In this analysis, the Stimulation Condition results were significant $\left(F_{(1,26)}=3.17 ; p=0.05\right)$, which suggested the presence of a different learning rate between the two groups (i.e., a lower rate with a-tDCS). Moreover, to evaluate the overall effects while considering the other conditions, we performed a one-way ANOVA with Stimulation Condition as a between-subjects factor. The result was not statistically significant $\left(F_{(5,72)}=1.03 ; p=0.32\right)$.

\section{RTs}

We performed a repeated-measures ANOVA with Block as a withinsubjects factor and Stimulation Condition and Gender as betweensubjects factors. These analyses showed a significant main effect for Block $\left(\varepsilon=0.74, p<0.001\right.$; Huynh-Feldt $F_{(4.44,319.36)}=25.94 ; p<$ $0.001)$ and $\operatorname{Gender}\left(F_{(1,72)}=7.31 ; p<0.01\right)$. The factor Stimulation Condition was not statistically significant $\left(F_{(5,72)}=1.03 ; p=0.40\right)$. Significant interactions between Block and Stimulation Condition $\left(F_{(30,432)}=2.12 ; p<0.001\right)$ and between Block, Stimulation Condition, and Gender $\left(F_{(30,432)}=1.79 ; p<0.001\right)$ were present, but post hoc comparisons (Bonferroni corrections) did not confirm their relevance.

Regarding the main effect for Block, multiple post hoc comparisons revealed a statistically significant difference between block 1 and the other blocks; between block 2 and all the other blocks but block 3; and between block 3 and blocks 6 and 7. As expected, the subjects were slower at the beginning of the task than at the end. The main effect for Gender showed that males were faster than females in task execution (mean RT \pm SEM: male $=682 \pm 27 \mathrm{~ms}$, female $=773 \pm 28 \mathrm{~ms}$ ).

\section{Sensations induced by different types of tES}

Only one recently published paper (Ambrus et al., 2010) has considered the skin perception thresholds for both tDCS and tRNS. Nevertheless, Ambrus et al. (2010) only compared the detection rates of the tDCS and tRNS and found that tRNS-induced sensations were less frequently perceived than sensations induced by tDCS. Based on these data, they proposed using tRNS application as a possible alternative to tDCS. In addition to analyzing the differences between the stimulation types, we also analyzed all of the possible induced sensations in detail. Each participant completed a questionnaire at the end of the experiment (Fertonani et al., 2010) and reported having tolerated the stimulation without discomfort. The results of the questionnaire are reported in Table 2. Participants were unable to distinguish the real stimulation from the placebo stimulation. A one-way ANOVA for each sensation was performed to compare the different stimulations. No difference between the tRNS and sham conditions was found. Interestingly, the analysis highlighted a statistically significant difference $(p<0.01)$ between both a-tDCS and c-tDCS and the other conditions with respect to itching. Furthermore, the a-tDCS was different $(p<0.01)$ from the other conditions with respect to irritation and burning. In general, the tDCS-induced sensations were perceived more strongly (Poreisz et al., 2007; Ambrus et al., 2010) than the tRNS- or sham-induced sensations. With tDCS, pain, heat, iron taste, and fatigue were comparable to the sham condition, but irritation, burning, and itching were perceived with real, but not sham, stimulation. In contrast, hf-tRNS and lf-tRNS were indistinguishable from sham conditions for all of the sensations examined. This characteristic makes tRNS an optimal tool for experimental designs in which sham stimulations should not differ from real stimulations. 
Table 2. Transcranial electrical stimulation-induced sensations: mean intensity of the sensations reported by subjects after tES, and the percentage of subjects who reported each sensation

\begin{tabular}{|c|c|c|c|c|c|c|c|c|}
\hline$\overline{\mathrm{tES}}$ & Irritation & Pain & Burning & Heat & Itch & Iron taste & Fatigue & Effect on performance \\
\hline \multicolumn{9}{|l|}{ hf-tRNS } \\
\hline $\begin{array}{l}\text { Intensity } \\
\text { Subjects }\end{array}$ & 0.1 & 0.0 & 0.1 & 0.3 & 0.3 & 0.0 & 0.5 & 0.4 \\
\hline$(\%)$ & 14 & 0 & 7 & 21 & 29 & 0 & 43 & 36 \\
\hline \multicolumn{9}{|l|}{ If-tRNS } \\
\hline $\begin{array}{l}\text { Intensity } \\
\text { Subjects }\end{array}$ & 0.0 & 0.1 & 0.0 & 0.0 & 0.4 & 0.2 & 0.1 & 0.1 \\
\hline$(\%)$ & 0 & 7 & 0 & 0 & 36 & 14 & 7 & 14 \\
\hline \multicolumn{9}{|l|}{ a-tDCS } \\
\hline $\begin{array}{l}\text { Intensity } \\
\text { Subjects }\end{array}$ & 1.1 & 0.2 & 0.7 & 0.4 & 1.4 & 0.1 & 0.4 & 0.5 \\
\hline (\%) & 79 & 14 & 50 & 29 & 86 & 7 & 29 & 36 \\
\hline \multicolumn{9}{|l|}{$c-t D C S$} \\
\hline $\begin{array}{l}\text { Intensity } \\
\text { Subjects }\end{array}$ & 0.6 & 0.1 & 0.3 & 0.1 & 1.4 & 0.1 & 0.2 & 0.1 \\
\hline$(\%)$ & 50 & 7 & 21 & 7 & 93 & 7 & 14 & 14 \\
\hline \multicolumn{9}{|l|}{ Sham } \\
\hline $\begin{array}{l}\text { Intensity } \\
\text { Subjects }\end{array}$ & 0.2 & 0.0 & 0.1 & 0.1 & 0.2 & 0.0 & 0.4 & 0.5 \\
\hline$(\%)$ & 21 & 0 & 7 & 14 & 14 & 0 & 29 & 29 \\
\hline \multicolumn{9}{|l|}{$\mathrm{Cz}$} \\
\hline $\begin{array}{l}\text { Intensity } \\
\text { Subjects }\end{array}$ & 0.3 & 0.0 & 0.1 & 0.1 & 0.4 & 0.1 & 0.1 & 0.3 \\
\hline (\%) & 29 & 0 & 7 & 14 & 36 & 7 & 7 & 21 \\
\hline
\end{tabular}

Sensation intensity was evaluated on a five-point scale: $0=$ none, $1=$ mild, $2=$ moderate, $3=$ considerable, and $4=$ strong. The column "Effect on performance" indicates the participants' subjective feelings relative to the tES-induced sensation's effect on performance.

Furthermore, we calculated, for all the experimental conditions, the correlation between the intensity of perceived sensations index (calculated for each subject as the sum of the values reported in the questionnaire for each sensation) and the mean $d^{\prime}$ values in the five blocks of real stimulation. The analysis was not statistically significant $\left(r_{(82)}=0.04, p>0.05\right)$. We also tested the correlation for the a-tDCS group because the subjects reported slightly higher perceptions of discomfort. Similarly, this analysis was not statistically significant $\left(r_{(12)}=-0.09, p>0.05\right)$. This result confirms the absence of a correlation between perceived discomfort and task performance.

\section{Control-hf-tRNS experiment}

The performance of the control-hf-tRNS group is depicted in Figure 4. A repeated-measures ANOVA on the six blocks of the task highlighted the main effect of Block $(p<0.01)$. Post hoc comparisons revealed that block 1 was different from blocks 2, 3, 4, 5, and 6, and blocks 2, 3, and 4 differed from blocks 5 and 6 .

Furthermore we compared the results of this group with the sham and hf-tRNS groups of the main experiment. We performed a repeated-measures ANOVA with Block (from 1 to 6) as a within-subjects factor and Stimulation Condition (control-hftRNS, hf-tRNS, and sham) as between-subjects factor. We observed a significant main effect for Block $\left(F_{(5,170)}=24.70 ; p<\right.$ $0.001)$ and Stimulation Condition $\left(F_{(2,34)}=5.59 ; p<0.01\right)$. Regarding the main effect of Block, post hoc comparisons revealed a statistically significant difference between block 1 and blocks 2 , $3,4,5$, and 6; between blocks 2, 3, and 4 and blocks 5 and 6; and between block 5 and block 6 .

Regarding the main effect of Stimulation Condition, as was expected post hoc comparisons revealed that control-hf-tRNS (mean $d^{\prime} \pm$ SEM $=0.614 \pm 0.142$ ) was significantly different from sham $(0.375 \pm 0.116)$. The difference between hf-tRNS $(0.770 \pm 0.130)$ and sham was also statistically significant.

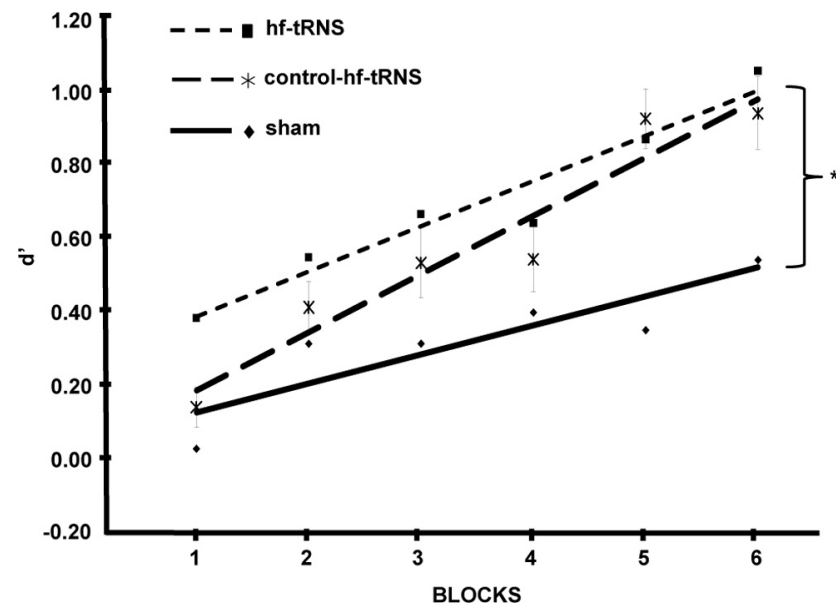

Figure 4. Control-hf-tRNS experiment results compared with main experiment hf-tRNS and sham groups. Data of control-hf-tRNS group are represented as the mean \pm SEM. The lines represent the fit of each condition: the thick dashed line corresponds to control-hf-tRNS, the thin dashed line corresponds to hf-tRNS, and the continuous thick line corresponds to sham. The asterisk near the curly bracket indicates the statistically significant differences between both control-hf-tRNS and sham $(p<0.05)$ and hf-tRNS and sham $(p<0.05)$.

Student's $t$ test revealed that in the first block the performance of control-hf-tRNS group was not different from sham $(t=$ $0.771, \mathrm{df}=21, p=0.45)$, whereas it was different from hf-tRNS $(t=-2.091, \mathrm{df}=21, p<0.05)$. In the last block, vice versa, the control-hf-tRNS performance was different from sham $(t=$ 2.556, $\mathrm{df}=21, p<0.05)$, but not from hf-tRNS $(t=-0.645$, $\mathrm{df}=21, p=0.53$ ).

To specifically evaluate learning rate differences for controlhf-tRNS versus hf-tRNS, a-tDCS, and sham, we performed a one-way ANOVA on the slope values with Stimulation Condition as a between-subjects factor. The result was statistically sig- 
nificant $\left(F_{(3,47)}=2.99 ; p<0.05\right)$. Post hoc comparisons revealed a statistically significant difference between control-hf-tRNS and both sham and a-tDCS groups. The difference between hf-tRNS and a-tDCS obtained in the main experiment was therefore confirmed.

These new results strengthen our main experiment data. As shown in Figure 4, the performance in the control-hf-tRNS group is very similar to the hf-tRNS one, except for the first block, in which the control-hf-tRNS performance is similar to the sham one.

\section{Discussion}

We observed an improvement in performance in the ODT when subjects were stimulated with hf-tRNS. With a-tDCS, we observed an initial facilitation, which was followed by a reduction of the learning phenomena. With c-tDCS and Cz stimulation, the performance was identical to the performance with sham stimulation. A not well defined role is that of lf-tRNS. With this stimulation, we observe an improvement, but the performance was not statistically different either from sham or from hf-tRNS.

Terney et al. (2008) reported a modulation of cortical excitability after $10 \mathrm{~min}$ of hf-tRNS to the primary motor cortex. The increase in MEP amplitude, which persisted after the end of the stimulation, was greater than the increase that is usually obtained with a-tDCS. Based on these results, Terney et al. (2008) proposed that tRNS induced a facilitatory effect similar to that of a-tDCS (Terney et al., 2008; Ambrus et al., 2010), even if it was sustained by different mechanisms. They also showed behavioral improvement in a motor learning task, but only when the full frequency spectrum (from 0.1 to $640 \mathrm{~Hz}$ ) was used, which does not allow for determination of whether a more restricted frequency range is responsible for the observed effect. For this reason, in our study, we applied the low and high frequencies separately.

Changes induced by tES are considered to be dependent on the NMDA receptor (e.g., Ridding and Ziemann, 2010). Shortand long-term tDCS effects are not observed after administration of an NMDA receptor antagonist or blocking $\mathrm{Na}^{+}$channels (Liebetanz et al., 2002; Nitsche et al., 2003b). On this basis, both LTP and its opposite, LTD, have been postulated to explain the persistent effects of brain stimulation on cortical activity (Thickbroom, 2007; Ziemann and Siebner, 2008; Nitsche et al., 2009; Fritsch et al., 2010). Nevertheless additional aspects should be considered in the relation to the differential stimulation characteristics and effects. Plasticity induced by different type of tES at the level of brain networks might be determined by changes that alter the property of synaptic plasticity. In the present study, we demonstrated that tRNS and a-tDCS have different behavioral effects, at least in the visual domain, which led us to conclude that the two types of stimulation are not fully interchangeable. In fact, the facilitation effect induced during the stimulation by tRNS was not present with a-tDCS. Terney et al. (2008) suggest that using tRNS sodium channels activity can be augmented. After a depolarization, repolarization of sodium channels would generally take some time, but if a repeated stimulation is applied these channels can be reopened in a shorter time (Schoen and Fromherz, 2008). Because tRNS is a repetitive, random, and subthreshold stimulation, we hypothesized that it would induce temporal summation of neural activity when the time constant of a neuron is sufficiently long to permit the summation of two stimuli presented in close sequence (i.e., the high-frequency range used in our experiment). These mechanisms should not be present with a-tDCS because such a polarizing stimulation may induce an initial facilitation, which could be followed by adaptations to rebalance the modulation of ion channel conductance. It might be likely that in the a-tDCS's initial phase, the cortical excitability shifted because of membrane polarization, and this shift induced a strengthening of the neural circuitry that improved performance. The initial increase in excitability, however, was followed by an adaptation of the neural system through mechanisms likely based on rebalancing the modulation of voltage-dependent ion channel conductance. The tDCS effects possibly relied mainly on the self-regulatory actions of voltage-dependent channels to induce inactivation during sustained depolarization (Levitan and Kaczmarek, 2002). Interestingly, some ion channels undergo a progressive decrease of activation in response to constant activation by a voltage change. In some cases, these voltage-dependent channels become inactive (i.e., closed) after an earlier activation, even if depolarization is maintained (Kurachi and Ishii, 2004). The inactivation of voltage-dependent channels is fundamental to determine whether there is a progressive decrease in the neuronal response to sustained exposure to a stimulus. This change in channel activity is termed the "rate of inactivation," and it is primarily related to faster sodium channels. Potassium channels also undergo inactivation, but the rate is much slower than sodium channels (Levitan and Kaczmarek, 2002). Importantly, potassium channels also participate in determining the actual rate at which a neuron fires, which makes them important contributors to the final outcome. Fast inactivation of calcium conductance during prolonged depolarization has also been reported in voltage-clamp studies of calcium currents (Eckert et al., 1977; Kostyuk and Krishtal, 1977).

This inactivation due to adaptation of ion channels may justify the absence of learning differences between the first and the last block of stimulation in the a-tDCS condition. Similar to our results, Antal et al. (2004b) found that a-tDCS over V5 only improved performance in the first block of a visuomotor learning task. Consistent with Antal et al. (2004b), the present results showed that a-tDCS immediately enhanced cortical excitability and ameliorated stimuli perceptions, but this effect disappeared in later blocks. We concluded that the dynamics of the induced effects differ between a-tDCS and tRNS because we found a difference in learning rates between the two types of stimulation.

Based on the homeostasis theory, we would expect c-tDCS to induce an initial performance deterioration followed by realignment to the normal trend of the sham group in subsequent blocks. In the present study, however, it was impossible to observe an impairment in performance because of a "floor effect" in the first block (i.e., it is impossible to have a performance lower than chance level). Therefore, the cathodal stimulation was completely ineffective from a behavioral perspective. The absence of a cathodal effect has also been reported in previous studies (Antal et al., 2004d; Fertonani et al., 2010; Kraft et al., 2010), although several papers have reported either inhibitory (Antal et al., 2001, 2003a,b, 2004a) or facilitatory (Antal et al., 2004c; Accornero et al., 2007) effects. This discrepancy is probably due to several factors, such as the different experimental tasks (e.g., patternreversal checkerboard vs sinusoidal luminance gratings) and the differences in stimulation parameters (e.g., intensity, duration, electrode size, and the location and direction of the current flow) (Nitsche et al., 2008). The placement of the reference electrode, which influences the direction and shunting of the current flow, seems particularly important. These aspects highlight the role of methodological differences in shaping the effects of tES (Antal and Paulus, 2008).

Importantly, the results obtained within the motor system are not always equivalent to the results obtained in the visual system 
or other areas. The cytoarchitectonic and myeloarchitectonic differences, including differences in neuron diameters, may explain the differential inductions observed in different regions. The principal feature of the motor cortex is the large pyramidal cells of the fifth layer of the Giant pyramidal cells of Betz (Brodal, 1981), which are not present in the visual areas. Neuronal threshold is inversely related to axon diameter; axons with a large diameter have a lower impedance, and large axons have a large membrane surface. This difference in structure means that changes in polarization may, in theory, be more "easily" induced over the motor cortex compared with other cortices because of the presence of large fibers. Nevertheless, there are some limitations related to the dendritic structure of pyramidal neurons (Stuart and Spruston, 1998; Spruston, 2008). In addition, the striate visual cortex is different from the precentral motor cortex; the former is granular and the latter is agranular. Indeed, the visual cortex has greater morphological variability (Dougherty et al., 2003), stellate cells are present instead of pyramidal neurons, and the striate cortex is characterized by its cortical thinness (Brodal, 1981). Together, these anatomical differences can explain the non-overlapping data for the same stimulation parameters over different cortices (Terney et al., 2008). Thus, for anatomical geometrical reasons and differences in current characteristics, hf-tRNS may induce a more robust effect than tDCS over the visual cortex.

Our most interesting finding was the broad performance enhancement that was obtained with tRNS. There are different mechanisms that can explain the induced behavioral effects and their dissimilarity to the effects induced by tDCS. In contrast to a-tDCS, we hypothesized that tRNS does not permit homeostasis of the system. Because of its particular wave shape, tRNS might induce temporal summation of small depolarizing currents, which could interact with the activity of the engaged neurons (Cash and Yuste, 1998) and enhance performance. Therefore, tRNS of neurons provides the driving force for a synaptic potentiation-like phenomenon. The effect was more pronounced with hf-tRNS, which was likely due to the frequency range applied $(100-640 \mathrm{~Hz})$. Because the time constant of the cell body and dendrites is between 1 and $10 \mathrm{~ms}$ (Kandel et al., 2000), stimulation between 100 and $1000 \mathrm{~Hz}$ may be optimal for affecting neuronal communication.

In the low-frequency range, the random stimulation effect was weaker and not statistically different from sham or from hf-tRNS which may be due to the presence of relatively high frequencies of stimulation (from 80 to $100 \mathrm{~Hz}$ ) at the upper end of this low-frequency range. These higher frequencies could interact analogously to hf-tRNS. This point should be clarified in future studies with the application of more specific frequency ranges (e.g., $40-60 \mathrm{~Hz}$ vs $80-100 \mathrm{~Hz}$ ). In this respect, studies have shown that different classes of neurons are activated at different frequencies (Freeman et al., 2010). Because different cortical areas contain different neuronal types, a specific band of frequencies might lead to the response of a subpopulation of neurons.

A further possible explanation for the effects induced by tRNS can be in the frame of the stochastic resonance phenomenon (e.g., Miniussi et al., 2010). tRNS is by definition a stimulation that induce nonfinalized random activity in the system i.e., noise. In general, noise decreases performance, but nonlinear systems, like the brain, can use noise to enhance performance through stochastic resonance (see Moss et al., 2004). The presence of neuronal noise might confer to neurons more sensitivity to a given range of weak inputs, i.e., those neurons "randomly activate" that go in the same direction as the signal, thereby rendering the noise in the signal. In this framework, it is possible to explain facilitatory results in terms of the relationship between noise and signal in the nervous system, so enhanced performance could be observed with an optimum level of noise (Antal et al., 2004c; Ruzzoli et al., 2010). Nevertheless, even if the term random noise stimulation can evocate such explanation, the present data do not allow us to draw a consideration in this framework since we cannot characterize the temporal coding between neural populations (i.e., neural synchronization or phase locking) by the present protocol.

In conclusion, the present data confirm the efficacy of hftRNS as a technique to improve performance in a visual perceptual learning task and show its superiority over tDCS in inducing facilitatory effects. We also demonstrated the high specificity of this stimulation over the V1 cortex, since hf-tRNS applied over the vertex, a brain area that is not involved in the ODT task, was totally ineffective, and subject performance was similar to performance after sham stimulation.

We suggest that the mechanism of action of tRNS is based on the repeated subthreshold stimulations that prevent homeostasis of the system. This effect might potentiate the activity of the neural populations involved in cognitive tasks that facilitate brain plasticity by strengthening synaptic transmission between neurons. Modulation of synaptic transmission efficacy can result in excitability and activity changes in specific cortical networks that are activated by the task's execution, and these changes correlate with cognitive plasticity at the behavioral level.

\section{References}

Accornero N, Li Voti P, La Riccia M, Gregori B (2007) Visual evoked potentials modulation during direct current cortical polarization. Exp Brain Res 178:261-266.

Ambrus GG, Paulus W, Antal A (2010) Cutaneous perception thresholds of electrical stimulation methods: comparison of tDCS and tRNS. Clin Neurophysiol 121:1908-1914.

Antal A, Paulus W (2008) Transcranial direct current stimulation and visual perception. Perception 37:367-374.

Antal A, Nitsche MA, Paulus W (2001) External modulation of visual perception in humans. Neuroreport 12:3553-3555.

Antal A, Kincses TZ, Nitsche MA, Paulus W (2003a) Manipulation of phosphene thresholds by transcranial direct current stimulation in man. Exp Brain Res 150:375-378.

Antal A, Kincses TZ, Nitsche MA, Paulus W (2003b) Modulation of moving phosphene thresholds by transcranial direct current stimulation of $\mathrm{V} 1$ in human. Neuropsychologia 41:1802-1807.

Antal A, Kincses TZ, Nitsche MA, Bartfai O, Paulus W (2004a) Excitability changes induced in the human primary visual cortex by transcranial direct current stimulation: direct electrophysiological evidence. Invest Ophthalmol Vis Sci 45:702-707.

Antal A, Nitsche MA, Kincses TZ, Kruse W, Hoffmann KP, Paulus W (2004b) Facilitation of visuo-motor learning by transcranial direct current stimulation of the motor and extrastriate visual areas in humans. Eur J Neurosci 19:2888-2892.

Antal A, Nitsche MA, Kruse W, Kincses TZ, Hoffmann KP, Paulus W (2004c) Direct current stimulation over V5 enhances visuomotor coordination by improving motion perception in humans. J Cogn Neurosci 16:521-527.

Antal A, Varga ET, Nitsche MA, Chadaide Z, Paulus W, Kovács G, Vidnyánszky Z (2004d) Direct current stimulation over MT+/V5 modulates motion aftereffect in humans. Neuroreport 15:2491-2494.

Brodal A (1981) Neurological anatomy in relation to clinical medicine, Ed 3. New York: Oxford UP.

Carmel D, Carrasco M (2008) Perceptual learning and dynamic changes in primary visual cortex. Neuron 57:799-801.

Cash S, Yuste R (1998) Input summation by cultured pyramidal neurons is linear and position-independent. J Neurosci 18:10-15.

Chaieb L, Antal A, Paulus W (2008) Gender-specific modulation of short- 
term neuroplasticity in the visual cortex induced by transcranial direct current stimulation. Vis Neurosci 25:77-81.

Dougherty RF, Koch VM, Brewer AA, Fischer B, Modersitzki J, Wandell BA (2003) Visual field representations and locations of visual areas V1/2/3 in human visual cortex. J Vis 3:586-598.

Eckert R, Tillotson D, Ridgway EB (1977) Voltage-dependent facilitation of $\mathrm{Ca} 2+$ entry in voltage-clamped, aequorin-injected molluscan neurons. Proc Natl Acad Sci U S A 74:1748-1752.

Fahle M, Poggio T (2002) Perceptual learning. Cambridge, MA: MIT Press.

Fertonani A, Rosini S, Cotelli M, Rossini PM, Miniussi C (2010) Naming facilitation induced by transcranial direct current stimulation. Behav Brain Res 208:311-318.

Freeman DK, Eddington DK, Rizzo JF 3rd, Fried SI (2010) Selective activation of neuronal targets with sinusoidal electric stimulation. J Neurophysiol 104:2778-2791.

Fritsch B, Reis J, Martinowich K, Schambra HM, Ji Y, Cohen LG, Lu B (2010) Direct current stimulation promotes BDNF-dependent synaptic plasticity: potential implications for motor learning. Neuron 66:198-204.

Gandiga PC, Hummel FC, Cohen LG (2006) Transcranial DC stimulation (tDCS): a tool for double-blind sham-controlled clinical studies in brain stimulation. Clin Neurophysiol 117:845-850.

Gilbert CD, Sigman M, Crist RE (2001) The neural basis of perceptual learning. Neuron 31:681-697.

Inghilleri M, Conte A, Currà A, Frasca V, Lorenzano C, Berardelli A (2004) Ovarian hormones and cortical excitability. An rTMS study in humans. Clin Neurophysiol 115:1063-1068.

Kanai R, Chaieb L, Antal A, Walsh V, Paulus W (2008) Frequencydependent electrical stimulation of the visual cortex. Curr Biol 18:18391843.

Kandel ER, Schwartz JH, Jessell MT (2000) Principles of neural sciences, Ed 4. New York: McGraw-Hill.

Kostyuk PG, Krishtal OA (1977) Effects of calcium and calcium-chelating agents on the inward and outward current in the membrane of mollusc neurones. J Physiol 270:569-580.

Kraft A, Roehmel J, Olma MC, Schmidt S, Irlbacher K, Brandt SA (2010) Transcranial direct current stimulation affects visual perception measured by threshold perimetry. Exp Brain Res 207:283-290.

Kuo MF, Paulus W, Nitsche MA (2006) Sex differences in cortical neuroplasticity in humans. Neuroreport 17:1703-1707.

Kurachi Y, Ishii M (2004) Cell signal control of the G protein-gated potassium channel and its subcellular localization. J Physiol 554:285-294.

Levitan IB, Kaczmarek LK (2002) The Neuron. Cell and molecular biology, Ed 3. New York: Oxford UP.

Li W, Piëch V, Gilbert CD (2004) Perceptual learning and top-down influences in primary visual cortex. Nat Neurosci 7:651-657.

Liebetanz D, Nitsche MA, Tergau F, Paulus W (2002) Pharmacological approach to the mechanisms of transcranial DC-stimulation-induced aftereffects of human motor cortex excitability. Brain 125:2238-2247.

Matthews N, Liu Z, Geesaman BJ, Qian N (1999) Perceptual learning on orientation and direction discrimination. Vision Res 39:3692-3701.

Miniussi C, Ruzzoli M, Walsh V (2010) The mechanism of transcranial magnetic stimulation in cognition. Cortex 46:128-130.

Moliadze V, Antal A, Paulus W (2010) Electrode-distance dependent aftereffects of transcranial direct and random noise stimulation with extracephalic reference electrodes. Clin Neurophysiol 121:2165-2171.

Moss F, Ward LM, Sannita WG (2004) Stochastic resonance and sensory information processing: a tutorial and review of application. Clin Neurophysiol 115:267-281.

Nitsche MA, Schauenburg A, Lang N, Liebetanz D, Exner C, Paulus W, Tergau F (2003a) Facilitation of implicit motor learning by weak transcranial direct current stimulation of the primary motor cortex in the human. J Cogn Neurosci 15:619-626.

Nitsche MA, Fricke K, Henschke U, Schlitterlau A, Liebetanz D, Lang N, Henning S, Tergau F, Paulus W (2003b) Pharmacological modulation of cortical excitability shifts induced by transcranial direct current stimulation in humans. J Physiol 553:293-301.

Nitsche MA, Cohen LG, Wassermann EM, Priori A, Lang N, Antal A, Paulus W, Hummel F, Boggio PS, Fregni F, Pascual-Leone A (2008) Transcranial direct current stimulation: State of the art 2008. Brain Stimul 1:206-223.

Nitsche MA, Kuo MF, Grosch J, Bergner C, Monte-Silva K, Paulus W (2009) D1-receptor impact on neuroplasticity in humans. J Neurosci 29:26482653.

Poreisz C, Boros K, Antal A, Paulus W (2007) Safety aspects of transcranial direct current stimulation concerning healthy subjects and patients. Brain Res Bull 72:208-214.

Ridding MC, Ziemann U (2010) Determinants of the induction of cortical plasticity by non-invasive brain stimulation in healthy subjects. J Physiol 588:2291-2304.

Rossi S, Hallett M, Rossini PM, Pascual-Leone A, the Safety of TMS Consensus Group (2009) Safety, ethical considerations, and application guidelines for the use of transcranial magnetic stimulation in clinical practice and research. Clin Neurophysiol 120:2008-2039.

Ruzzoli M, Marzi CA, Miniussi C (2010) The neural mechanisms of the effects of transcranial magnetic stimulation on perception. J Neurophysiol 103:2982-2989.

Sale MV, Ridding MC, Nordstrom MA (2007) Factors influencing the magnitude and reproducibility of corticomotor excitability changes induced by paired associative stimulation. Exp Brain Res 181:615-626.

Schiltz C, Bodart JM, Dubois S, Dejardin S, Michel C, Roucoux A, Crommelinck M, Orban GA (1999) Neuronal mechanisms of perceptual learning: changes in human brain activity with training in orientation discrimination. Neuroimage 9:46-62.

Schoen I, Fromherz P (2008) Extracellular stimulation of mammalian neurons through repetitive activation of $\mathrm{Na}+$ channels by weak capacitive currents on a silicon chip. J Neurophysiol 100:346-357.

Schoups A, Vogels R, Qian N, Orban G (2001) Practising orientation identification improves orientation coding in V1 neurons. Nature 412:549 553.

Shiu LP, Pashler H (1992) Improvement in line orientation discrimination is retinally local but dependent on cognitive set. Percept Psychophys 52:582-588.

Smith MJ, Keel JC, Greenberg BD, Adams LF, Schmidt PJ, Rubinow DA, Wassermann EM (1999) Menstrual cycle effects on cortical excitability. Neurology 53:2069-2072.

Smith MJ, Adams LF, Schmidt PJ, Rubinow DR, Wassermann EM (2002) Effects of ovarian hormones on human cortical excitability. Ann Neurol 51:599-603.

Spruston N (2008) Pyramidal neurons: dendritic structure and synaptic integration. Nat Rev Neurosci 9:206-221.

Stuart G, Spruston N (1998) Determinants of voltage attenuation in neocortical pyramidal neuron dendrites. J Neurosci 18:3501-3510.

Terney D, Chaieb L, Moliadze V, Antal A, Paulus W (2008) Increasing human brain excitability by transcranial high-frequency random noise stimulation. J Neurosci 28:14147-14155.

Thickbroom GW (2007) Transcranial magnetic stimulation and synaptic plasticity: experimental framework and human models. Exp Brain Res 180:583-593.

Thiele A (2004) Perceptual learning: is V1 up to the task? Curr Biol 14:R671-R673.

Ts'o DY, Gilbert CD, Wiesel TN (1986) Relationships between horizontal interactions and functional architecture in cat striate cortex as revealed by cross-correlation analysis. J Neurosci 6:1160-1170.

Vogels R, Orban GA (1985) The effect of practice on the oblique effect in line orientation judgments. Vision Res 25:1679-1687.

Yacoub E, Harel N, Ugurbil K (2008) High-field fMRI unveils orientation columns in humans. Proc Natl Acad Sci U S A 105:10607-10612.

Ziemann U, Siebner HR (2008) Modifying motor learning through gating and homeostatic metaplasticity. Brain Stimul 1:60-66. 